\title{
Instrumentos de evaluación en rehabilitación psicosocial
}

\section{Assessment in psychosocial rehabilitation}

\begin{abstract}
RESUMEN: Se realiza una revisión de los instrumentos de evaluación disponibles en castellano en seis áreas: rehabilitación psicosocial general, necesidades, discapacidad, calidad de vida, psicopatología y carga familiar.

PALABRAS CLAVE: evaluación, rehabilitación psicosocial, esquizofrenia, escalas.
\end{abstract}

\begin{abstract}
The paper reviews all the assessment instruments available in Spanish in six areas: general psychosocial rehabilitation, needs, disability, quality of life, psychopathology and family burden.

KEYWORDS: assessment, psychosocial rehabilitation, schizophrenia, scales.
\end{abstract}

\section{Introducción.}

Los servicios de rehabilitación psicosocial atienden a personas que padecen trastornos mentales severos y crónicos. El objetivo de estos servicios es conseguir el máximo grado de recuperación del individuo. Una definición operativa de la recuperación en la esquizofrenia (1) válida asimismo para los trastornos severos y crónicos, seria entenderla como: remisión sintomática, participación plena o parcial en una tarea ocupacional o en un empleo o actividad formativa, una vida independiente sin la supervisión de familiares o cuidadores sustitutos, no depender totalmente de ayudas económicas por discapacidad y tener amigos con los que realizar actividades de forma regular.

En función de la evaluación realizada en cada paciente y el grado de recuperación conseguido, la asistencia realizada por los servicios de rehabilitación es mayor o menor. Actualmente existe un gran número de pacientes cuya atención en los servicios de rehabilitación comunitaria es posiblemente mayor de la que cabría esperar si se hubiera realizado una atención precoz, rápida, integral y priorizando la recuperación ante un primer brote o en la fase inicial de la enfermedad. Numerosos estudios están enfatizando la importancia de la detección y atención precoz (2). Asimismo, la evaluación funcional del paciente es un área que debe potenciarse a fin de poder obtener mayor información sobre las áreas a intervenir y sobre la efectividad de las intervenciones en el área de la rehabilitación (3).

La utilización de escalas, cuestionarios y tests que nos permitan obtener 
una información fiable, estandarizada y comparable con otras personas que presentan el mismo, y en algunos casos, otros trastornos mentales (4) se hace imprescindible en el área de la rehabilitación psicosocial. La evaluación inicial de la persona que entra en un Programa Individual de Rehabilitación y Reinserción y el seguimiento de su evolución, es uno de los estándares que deben plantearse los servicios; ya que nos permite evaluar el estado de cada una de las áreas susceptibles de ser intervenidas. Así mismo nos permitirá evaluar la eficacia de las intervenciones realizadas. Por otro lado, toda la información recogida a lo largo de las distintas intervenciones terapéuticas, nos permite revisar la eficacia y la eficiencia los programas de rehabilitación que se han desarrollado.

En este sentido es interesante poder realizar una aproximación a los instrumentos disponibles, así como las características y la utilización de estos en la práctica clínica.

Es importante tener en cuenta que un gran número de las escalas que se han revisado en el presente artículo, y que se utilizan en la valoración de programas de rehabilitación, fueron diseñadas para la evaluación de personas con esquizofrenia, debido a la alta prevalencia del trastorno en los servicios de rehabilitación. Posteriormente, algunas de estas escalas se han utilizado e incluso validado para otros trastornos mentales severos.

\section{Evaluación general en rehabilitación psicosocial}

Existen una serie de instrumentos validados para personas con trastornos mentales severos que nos permiten evaluar el estado de estos en su totalidad, teniendo en cuenta sus capacidades o limitaciones, su estado psicopatológico y sus relaciones sociales.

La escala SCHIZOM (5) es una entrevista semiestructurada que evalúa proceso y déficits asociados a la esquizofrenia a lo largo del tiempo. Tiene 3 versiones distintas: línea base usuario, línea base informador y seguimiento (4-6 meses). Evalúa las siguientes áreas: ajuste previo a la enfermedad, historia familiar de enfermedad mental, síntomas recientes, funcionamiento cognitivo, actividades de ocio, actividades instrumentales de la vida diaria, actividades sociales, vivienda, actividad educativa, actividad laboral, abuso de sustancias, problemas legales, violencia y suicidio, adherencia a la medicación, adherencia a la intervención psicosocial, apoyo familiar, uso de servicios, y los componentes del tratamiento: funcionamiento general introduciendo su evaluación con la escala de calidad de vida SF-36. La duración de la entrevista es de 30-35 minutos, para cada uno de los informantes. Su principal ventaja es que evalúa todos los dominios identifica- 
ORIGINALES Y REVISIONES

dos de la esquizofrenia: estado clínico, funcionamiento social, satisfacción vital y sensación de bienestar y bienestar en la comunidad. Presentó una validación concurrente y de sensibilidad al cambio fuerte, así como una consistencia interna asegurada para 3 de las escalas, y el $\alpha$ de Cronbach es moderada para 2 de las escalas y débil para las demás, en la versión original. Está traducida y adaptada al castellano al castellano por M. Muñoz y otros (Facultad de psicología. Universidad complutense de Madrid), pendiente de su validación.

La escala HONOS (6), traducida y validada al Castellano por Uriarte et al en 1998 (7), es un instrumento de evaluación desarrollado por la Unidad de Investigación del Royal Collage of Psychiatrists británico en el marco del Proyecto Health of the Nation del Departamento de Salud británico. Está constituida por un conjunto de escalas diseñadas para medir todo el rango de problemas físicos, personales y sociales asociados a la enfermedad mental y diseñada para la evaluación de adultos en contacto con servicios de asistencia secundaria y terciaria de Salud Mental. Puede ser utilizado por diferentes profesionales: psiquiatras, psicólogos, personal de enfermería y trabajadores sociales. Es un instrumento de evaluación clínica. Contiene 12 ítems que abarcan cuatro áreas: problemas conductuales (agresividad, auto agresión, uso de substancias), deterioro (disfunción cognitiva y discapacidad física), problemas clínicos (depresión, alucinaciones, ideas delirantes y otros síntomas) y problemas sociales (funcionamiento general, alojamiento y problemas ocupacionales). Presenta limitaciones ya que no debe usarse en la atención primaria y no se concibió específicamente para evaluar costes, estadísticas de dispositivos asistenciales, o el uso del tiempo de los profesionales, pero puede emplearse como parte de la evaluación de estos aspectos si se utiliza combinado con otras herramientas.

Una de las escalas especialmente útil en la evaluación de enfermos mentales crónicos es la escala REHAB $(8,9)$ desarrollada para personas institucionalizadas pero que puede también ser usada para evaluar pacientes que viven en el hospital, que asisten a un centro de rehabilitación psicosocial o que viven en casas residenciales. Es una escala que ha demostrado ser fiable y válida (10). Puede ser utilizada para: Identificar pacientes institucionalizados con potencial para vivir en la comunidad, realizar una medida general de la discapacidad en grupos de pacientes con problemas similares, ayudando por tanto para la composición de unidades, planificar el programa de rehabilitación y establecer una línea base y evaluar los cambios en la conducta del usuario en un tiempo dado y detectar las áreas más significativas de intervención.

La REHAB está formada por 23 ítems en total que conforman dos subescalas: la escala de Conductas Desviadas, que evalúa la frecuencia con que se presentan conductas difíciles o perturbadoras (incontinencia, abuso verbal y físico, auto agresión, conducta sexual escandalosa, salidas no previstas y hablar/reír a 
solas) y la escala de Conducta General, el contenido de sus ítems hace referencia a características de la comunicación, interacción social, auto cuidado, actividad social y utilización de diversos servicios públicos.

\section{Evaluación de necesidades}

La evaluación de las necesidades de las personas que padecen un trastorno mental severo se ha convertido en un área imprescindible de detección, ya que determina el nivel de ayuda que necesitan para diferentes ámbitos de su vida; así como quién está facilitando este apoyo.

Las necesidades individuales se han evaluado con diferentes instrumentos, tales como el Needs for Care Assessment Schedule (NFCAS) (11), el Cardinal Needs Schedule (12) y el Camberwell Assessment of Need (CAN) (12). De todos ellos únicamente el CAN, diseñado en el Institute of Psychiatry Health Services(PRISM) de Londres, está validado en población española, por el grupo de Granada Sur (14). Los resultados obtenidos fueron una alta concordancia entre evaluadores y unas puntuaciones óptimas en la valoración test-retest, pero detectaron que el instrumento era más sensible si existe un conocimiento del paciente evaluado, por lo tanto, más útil en la versión clínica. También ha sido traducido y validado en diferentes idiomas, además del inglés, como son el sueco (15), italiano (16), holandés y danés $(17,18)$, presentando óptimas características psicométricas.

La novedad que presenta el CAN es la doble valoración de cada una de las necesidades por parte del usuario y del profesional, así que podemos decir que el instrumento son en realidad dos escalas independientes. Esto permite realizar las valoraciones de uno de los dos evaluadores en función de los objetivos del estudio propuesto.

El CAN consta de 22 áreas de necesidades: alojamiento, alimentación, cuidado del hogar, cuidado personal, actividades diarias, salud física, síntomas psicóticos, información, angustia, seguridad en sí mismo, seguridad en los otros, alcohol, drogas, compañía, relaciones de pareja, sexualidad, cuidado de los hijos, educación básica, teléfono, transporte, dinero y ayudas sociales. De cada una de estas áreas se valora la presencia o ausencia de necesidad. En el caso de que exista una necesidad ya sea grave o moderada con ayuda se valorará la ayuda informal y formal que recibe la persona y si la ayuda formal recibida es la adecuada. Cuando se habla de ayuda formal se refiere a que son los servicios sanitarios y/o sociosanitarios los que facilitan la ayuda para cubrir la necesidad presente. Respecto a la ayuda informal, se refiere a que es la familia o los amigos los que están facilitando la ayuda para cubrir la presencia de una determinada necesidad. 
ORIGINALES Y REVISIONES

Existen dos versiones del CAN, relativamente semejantes, para la utilización clínica y para la investigación. Por este motivo es un cuestionario útil tanto para la práctica diaria como para la evaluación de las necesidades desde la investigación. Además existe una versión abreviada (CANSAS) que consiste en evaluar únicamente la primera pregunta del cuestionario (presencia de necesidad, cubierta o no cubierta) en cada una de las necesidades, por el profesional y por el usuario $(19,20)$.

El 2-COM (21), es un cuestionario basado en el CAN para la evaluación de las necesidades. Se seleccionaron las necesidades más frecuentes encontradas en una muestra de 707 personas con problemas de salud mental que fueron evaluadas en tres tiempos durante dos años. Los ítems que evalúa son: alojamiento, cuidado del hogar, cuidado personal, actividades diarias, salud física (incluyendo problemas con la medicación y con los efectos secundarios), síntomas psicóticos, información, angustia (incluyendo dificultades para dormir, para olvidar cosas, sentirse cansado, capacidad de disfrute con lo que hace, sentirse tenso y sentirse preocupado) compañía, relaciones de pareja, sexualidad, transporte, dinero y ayudas sociales. Se les pregunta a los usuarios por cada una de las áreas si es un problema para ellos y si querían hablar de este problema.

Evaluación de discapacidad, el funcionamiento psicosocial y las actividades diarias.

La enfermedad mental crónica cursa con un deterioro que se evidencia a través de diversas discapacidades, referidas fundamentalmente a la pérdida de habilidades para el adecuado desempeño de roles sociales. La discapacidad se define como la inhabilidad o limitación para realizar tareas esperadas de un individuo dentro de un ambiente social (por ejemplo, auto cuidado, autonomía, afrontamiento) (22). En general, se pueden articular los déficits en las siguientes grandes áreas, que deben ser tenidas en cuenta al valorar la severidad de la pérdida de desempeño social: autocuidados, autonomía, autocontrol, relaciones interpersonales, ocio y tiempo libre y funcionamiento cognitivo.

El concepto de discapacidad y funcionamiento en realidad serían las dos caras de la misma moneda, dependiendo del lado en el que hagamos la evaluación. Sin embargo, cuando nos referimos a las actividades de la vida diaria, se está haciendo referencia a un subapartado del funcionamiento o de la discapacidad. Las escalas de evaluación, en la mayoría de los casos, no delimitan de forma estricta estos conceptos. Por todo ello, se hace referencia inicialmente a instrumentos de valoración de la discapacidad en general, para citar asimismo aquellas escalas que se centran en aspectos más concretos. 
Centrándonos en las escalas de evaluación de la discapacidad, la Organización Mundial de la Salud (OMS) ha desarrollado el WHODAS II, instrumento de medida del funcionamiento y la discapacidad conceptualmente compatible con la revisión reciente de la Clasificación Internacional del Funcionamiento, de la Discapacidad y de la Salud (CIF) de la OMS en 2001 (23) Se trata de una revisión completa de la WHODAS (publicada por la OMS en 1988) que refleja el pensamiento actual de la OMS sobre el funcionamiento y la discapacidad. Evalúa el funcionamiento diario en seis áreas de actividad desde una doble perspectiva, cuantitativa y de interferencia de vida: comprensión y comunicación, capacidad para moverse en su alrededor/entorno, cuidado personal, capacidad de relación con otras personas, actividades de la vida diaria y participación en la sociedad. Proporciona un perfil objetivo de funcionamiento en las seis áreas y la percepción subjetiva del paciente sobre el impacto que le producen las dificultades en cada una de las áreas. La WHODAS II evalúa discapacidad y déficits específicos y no incorpora síntomas psiquiátricos. Añadir, que la CIE-10 propone la utilización de la escala en su versión abreviada (DAS-S) (24). Este sencillo instrumento diseñado para registrar la valoración clínica de las discapacidades causadas por trastornos mentales, es una entrevista semiestructurada, donde las puntuaciones deben de estar basadas en el juicio clínico, tras la información obtenida de familias, informantes o documentos clínicos y de la propia observación. La escala de la OMS (WHO DAS-S) se acompaña de una serie de instrucciones para su correcta utilización, incluyendo una breve definición de los contenidos específicos de las áreas de funcionamiento, un listado de aspectos importantes de discapacidades específicas a tener presentes en la evaluación y puntuación y unas preguntas a modo de guía para la exploración de áreas específicas de funcionamiento.

En esta línea también podemos encontrar la DASII de Schubart y cols (25). Existe una adaptación española del DAS-II realizada por Montero y cols. (26) y Montero y Gómez-Beneyto (27). Evalúa el ajuste social del paciente esquizofrénico teniendo en cuenta su propio contexto social y cultural. Mide 5 áreas: comportamiento general (cuidado personal, empleo del tiempo libre, velocidad de la ejecución física y mental y capacidad de iniciativa para comunicarse socialmente), ejecución del rol social (vida familiar, relación de pareja, rol paternal, relaciones sexuales, relaciones sociales y trabajo), comportamiento del paciente en el hospital (si ha estado ingresado dos o más semanas en el último mes), factores modificantes (inquietudes personales para mejorar la situación, riesgos específicos, ambiente en el hogar -se revisan aspectos relacionados con la expresividad emocional del familiar clave informante- y apoyos sociales de que dispone y usa) y evaluación global.

La entrevista se realiza a una persona cercana al paciente y se centra en el último mes. La evaluación se basa en el juicio clínico del entrevistador, se com- 
ORIGINALES Y REVISIONES

para el funcionamiento del paciente con el funcionamiento normal o promedio de una persona de su mismo sexo, edad y ambiente sociocultural, lo que le confiere la característica de ser aplicable en diferentes ámbitos socio-culturales.

Las ventajas principales de la escala son el incluir un área que mide la importancia de los factores ambientales y familiares que inciden en el curso de la enfermedad y la introducción de un criterio comparativo actual con el que el paciente presentaba el año anterior, lo que permite valorar la evolución de cada uno de los ítems por separado. Al mismo tiempo puede ser útil en la planificación de las intervenciones al obtener de forma independiente el grado de disfunción en cada una de las áreas. Siendo la mayor dificultad el hecho que pueda considerarse subjetivo el criterio del clínico a la hora de valorar la proximidad o no a la norma.

La American Psychiatric Association propone la Escala de Evaluación de la Actividad Global EEAG (28) para la valoración del funcionamiento general de los pacientes a lo largo de un continuum de salud-enfermedad. Esta escala está siendo validada por un grupo de trabajo de la Fundació Seny. El único ítem de la escala, actividad global del paciente, se puntúa mediante una escala que oscila entre 100, actividad satisfactoria, y 1, expectativa manifiesta de muerte. Una de las críticas que recibe este instrumento es que en los criterios operativos de puntuación incorpora síntomas junto con la discapacidad, asimismo es una medida global y no específica de áreas de discapacidad, con lo cual, su utilización es limitada y aporta poca información relevante del paciente.

La escala LSP-Life Skills Profile (Perfil de Habilidades de la Vida Cotidiana) de Rosen y cols. (29) ha sido diseñada para medir el nivel de funcionamiento y de incapacidad del enfermo esquizofrénico. Ha sido traducida y validada con población española con patología psicótica activa y residual por Bulbena Vilarrasa y colaboradores (30), ya que la escala, al no incluir síntomas o conductas específicas de la esquizofrenia, ha resultado de utilidad con otro tipo de patologías mentales de larga evolución. La LSP está formada por 39 ítems y pretende medir el nivel de funcionamiento de enfermos mentales crónicos en situaciones y tareas comunes. Se compone de cinco subescalas: Auto Cuidado, Comportamiento Social Interpersonal, Comunicación-Contacto. La evaluación se realiza a partir de las informaciones aportadas por familiares o profesionales que conozcan bien a la persona evaluada. La escala ha demostrado buenos requisitos psicométricos de fiabilidad y validez (31).

La evaluación del funcionamiento psicosocial nos permite valorar el nivel de adaptación de la persona a la comunidad en relación con las Actividades de la Vida Diaria (AVD). Es de gran utilidad para poder trabajar sobre aquellas áreas en las que exista una mayor problemática y mayor repercusión en su vida cotidiana. 
La escala BELS (32) permite evaluar las actividades de la vida diaria en las personas que padecen un trastorno mental severo que están hospitalizadas y van a pasar a vivir en la comunidad. Ha sido traducida por el Grupo Andaluz de Investigación en Salud Mental, pero en esta versión no existen las puntuaciones de corte. Evalúa tanto el grado de oportunidad para la realización de una actividad de forma independiente como el nivel de realización de esa misma conducta o habilidad. Las áreas de evaluación son auto cuidado, habilidades domésticas (habilidades comunitarias, actividades y relaciones sociales). La información se obtiene de un informante que conozca al usuario; y el tiempo de observación es durante el mes previo.

La ILSS, Independenting Living Skills Survey (33), evalúa la capacidad para las AVD de los pacientes que viven en comunidad, residen con familiares o en centros especializados. Está compuesta por 112 items que se agrupan en 9 áreas: hábitos de alimentación, higiene y aseo personal, actividades domésticas, habilidades para prepararse la comida, auto cuidado de la propia salud, autoadministración económica, uso del transporte publico, ocio y tiempo libre y habilidades de búsqueda de empleo. Tiene una escala adicional que mide el grado en que cada conducta es problema lo que permite obtener información de las áreas prioritarias de intervención y estructurar un plan concreto en el proceso de rehabilitación, pero debe complementarse con aspectos relacionados con habilidades sociales y ajuste social.

El cuestionario lo cumplimentan personas cercanas al paciente y la evaluación se centra en el mes anterior al momento de la cumplimentación.

En castellano existe la adaptación de Fernández Larrinoa y cols de 1994 (34). Los coeficientes $\alpha$ varían entre 0.67 y 0.84 y la fiabilidad entre 0.63 y 0.89 . Las áreas de hábitos de alimentación, habilidades de cocina y habilidades para buscar trabajo presentan una fiabilidad menor debido a la falta de oportunidad para realizar estas conductas. La validez concurrente, realizada con el NOSIE-30 y el MACC-II es significativa.

La escala SFS de Birchwood y cols. (35) está diseñada para su uso en programas de intervención familiar con pacientes esquizofrénicos, intentando cubrir el vacío existente en la evaluación del funcionamiento social como variable en dichos programas. Está validada al castellano por Torres y Olivares (36). La escala evalúa los aspectos que se juzgan fundamentales a la hora de mantener al paciente en la comunidad. Se incluyen siete áreas: interacción y retraimiento social, conductas interpersonales, actividades pro-sociales, actividades de ocio, independencia-competencia, independencia-actividad y empleo-ocupación. Diferencia entre la falta de competencia para realizar una tarea para una vida independiente y la falta de la realización correcta. Es una entrevista que enumera las habilidades básicas, las conductas sociales, etc. que los informadores, en este 
ORIGINALES Y REVISIONES

caso familiares, puntúan como presentes o ausentes evitando juicios evaluativos. Los entrevistadores requieren un entrenamiento breve en su utilización. Se puede utilizar el instrumento de manera auto-aplicada.

La evaluación del funcionamiento social se ha convertido actualmente en un área de gran interés, por la demostrada eficacia de los programas de entrenamiento en habilidades sociales (33). La Escala Multidimensional de Expresión Social - Parte Motora (EMES-M) y la Escala Multidimensional de Expresión Social - Parte Cognitiva (EMES-C) (37), fueron diseñadas por Vicente Caballo a finales de la década de los ochenta con el fin de evaluar Habilidades Sociales. La EMES-M pretende medir el comportamiento del individuo de acuerdo con una serie de habilidades sociales específicas. La EMES-M consta de 64 ítems, en cada uno de los cuales se indica la frecuencia de aparición de conductas sociales, en una escala tipo Likert de 0 a 4 puntos. Ofrece información acerca de 12 diferentes factores que agrupan las siguientes situaciones: iniciación de interacciones, hablar en público/enfrentarse con superiores, defensa de los derechos del consumidor, expresión de molestia, desagrado, enfado, expresión de sentimientos positivos hacia el sexo opuesto, expresión de molestia y enfado hacia familiares, rechazo de peticiones provenientes del sexo opuesto, aceptación de cumplidos, tomar la iniciativa en las relaciones con el sexo opuesto, hacer cumplidos, preocupación por los sentimientos de los demás y expresión de cariño hacia los padres. Las puntuaciones más bajas indican peores puntuaciones en habilidades sociales.

En el libro de Caballo (38) aparecen las medias y desviaciones típicas de una muestra de estudiantes. La escala fue rellenada por 673 sujetos de tres universidades españolas: Madrid, Granada y Santiago de Compostela. Se han hallado los datos sobre su consistencia interna, su fiabilidad test-retest y su validez concurrente. Igualmente, la escala ha sido analizada factorialmente, dando como resultado 12 factores claramente definidos que explicaban el $48 \%$ de la varianza total. Varios de estos factores se refieren a clases específicas de personas, apoyando la posición de especificidad situacional con respecto a las habilidades sociales.

La escala EMES-C tiene como objetivo medir la frecuencia de pensamientos negativos ante diversas situaciones sociales. Consta de 44 ítems, en los que se indica su frecuencia de aparición en una escala tipo Likert de 0 a 4 puntos. Ofrece información acerca de 12 diferentes factores que agrupan las siguientes situaciones: temor a la desaprobación de los demás al expresar sentimientos negativos y al rechazar peticiones; temor a hacer y recibir peticiones, temor a hacer y recibir cumplidos, preocupación por la expresión de sentimientos positivos y la iniciación de interacciones con el sexo opuesto, temor a la evaluación negativa por parte de los demás en la expresión de conductas positivas, temor a una 
conducta negativa por parte de los demás en la expresión de conductas positivas, preocupación por la expresión de los demás en la expresión de sentimientos, preocupación por la impresión causada en los demás, temor a expresar sentimientos positivos, temor a la defensa de los derechos y asunción de posibles carencias propias. Cuanto mayor sea la puntuación en este cuestionario, mayor incidencia de los pensamientos distorsionados. Caballo y Ortega (39) describen los diversos ítems que comprende cada factor de la escala y, cómo obtener la media de la puntuación de un determinado sujeto en el factor que nos interese.

Estos instrumentos se encontraban validados con población universitaria española. A pesar de ello, se han utilizado con pacientes psicóticos (40, 41), pero sería interesante evaluar sus capacidades psicométricas en población con trastorno mental severo.

El Inventario de Asertividad (42) Este inventario consta de 40 situaciones sociales e interpersonales que de alguna manera exigen una respuesta de asertividad por parte del individuo. Tiene dos escalas: una se refiere al grado de malestar o ansiedad ante cada una de esas situaciones y otra a la probabilidad por parte del sujeto de responder asertivamente ante ellas. Esta diseñado para evaluar las habilidades sociales y conducta asertiva en población general aunque es utilizada con frecuencia en población con trastorno mental severo. Este cuestionario fue adaptado al castellano por Carrobles, Costa y del Ser.

\section{Calidad de Vida}

Desde la aparición de los neurolépticos, la psiquiatría ha pasado a preocuparse no únicamente de controlar y tratar de eliminar la sintomatología clínica, sino también a conseguir una mejor "calidad de vida" para los pacientes.

La Organización Mundial de la Salud (43) define calidad de vida como "la percepción personal de un individuo de su situación en la vida, dentro del contexto cultural y de valores en que vive, y en relación con sus objetivos, expectativas, valores e intereses".

En el campo de las esquizofrenias el tema de la calidad de vida aparece con gran impulso con Malm y cols. (44). A partir de esta fecha se produce un espléndido desarrollo y se elaboran varios modelos conceptuales, escalas de medida y trabajos empíricos sobre la calidad de vida en estas enfermedades. Pero en "las esquizofrenias el tema de la calidad de vida es especialmente complejo por las características clínicas de estas enfermedades, (...) por la necesidad de conceptuar y definir correctamente si algunos síntomas negativos deben evaluarse como verdaderos síntomas, en el sentido clínico tradicional, o bien si son ya conse- 
ORIGINALES Y REVISIONES

cuencia de la reacción del paciente ante una enfermedad y el ambiente familiar y social y también en qué medida son consecuencia de la medicación administrada crónicamente" (45).

Un reflejo de las dificultades que entraña el tratamiento de la calidad de vida es la gran cantidad de escalas de evaluación; debido a las diferencias conceptuales sobre qué se entiende por calidad de vida, temporalidad y durabilidad. A continuación hacemos referencia de una representación significativa de algunas de ellas, centrándonos en aquellas que consideramos que pueden ser de utilidad en el diagnóstico y tratamiento del paciente psicótico.

Las escalas que evalúan la calidad de vida relacionada con la salud son el Cuestionario SF-36 y el Cuestionario EQ-5D. El Cuestionario SF-36 sobre el Estado de Salud (Short-Form, SF-36) de Ware y Sherbourne (46). El SF-36 evalúa el nivel de calidad de vida relacionada con la salud, conceptualmente basado en un modelo bidimensional de salud física y mental. Es un instrumento validado al castellano (47). Consta de 36 ítems que se agrupan en 8 escalas: funcionamiento físico (FF), rol físico (RF), dolor (D), salud general (SG), vitalidad (V), funcionamiento social (FS), rol emocional (RE) y por último salud mental (SM). Las 8 escalas se agrupan a su vez en dos medidas sumarias: salud física y salud mental. Las escalas que forman la medida sumaria de salud física son: FF, RF, D, SG y V. Las escalas que forman la medida sumaria de salud mental son: SG, V, FS, RE y SM. Es una escala autoaplicada y tiene el marco de referencia temporal en el momento actual.

El Cuestionario de Salud EQ-5D (48) está diseñado con la intención de obtener un instrumento estandarizado y genérico para la evaluación de la calidad de vida relacionado con la salud, que permita el intercambio de datos entre los diferentes países. Evalúa en una primera parte las variables de movilidad, cuidado personal, actividades cotidianas, dolor/malestar y ansiedad depresión. En la segunda parte pide al paciente la percepción que tiene sobre su estado de salud (peor estado de salud imaginable al mejor estado de salud imaginable). El marco de referencia temporal es el día de la entrevista. Existe una versión en castellano validada por Badía et al $(49,50,51)$.

Por otro lado podemos identificar otro tipo de cuestionarios que tienen por objeto de evaluación de la calidad de vida, la percepción que tiene el individuo en su día a día. Dentro de este marco podemos identificar la WHOQOL-100, de la OMS, y la Q-LES-Q.

El Instrumento de Evaluación de Calidad de Vida de la Organización Mundial de la Salud (World Health Organization Quality of Life, WHOQOL-100) (52) es una escala autoaplicada que consta de 100 ítems. Este instrumento determina la percepción de los individuos de su situación en la vida dentro del contexto cultural y del sistema de valores en el que viven, y en relación a sus objetivos, 
expectativas e intereses. Esta medida se realiza basándose en la percepción del sujeto y en su valoración sobre diversos aspectos de su vida. El WHOQOL100 está estructurado de forma jerárquica y cuenta con una evaluación global de la calidad de vida y evaluaciones en 6 distintos dominios, que a su vez están integrados por 24 distintas facetas: físico, psicológico, nivel de independencia, relaciones sociales, entorno y espiritualidad/religión/creencias personales.

La OMS ha desarrollado una versión abreviada, el WHOQOL-BREF formado por 26 ítems ( 2 ítems generales y 1 ítem por cada una de las 24 facetas que valora). Esta versión sólo proporciona información sobre 4 de las 6 dimensiones del WHOQOL-100: física, psicológica, relaciones sociales y entorno. De esta versión existe una versión en castellano editada por R. Lucas (53).

El Q-LES-Q (Quality of Lofe Enjoyment and Satisfaction Questionnaire, Q-LES-Q) $(54,55)$ fue desarrollado con la intención de obtener un cuestionario que midiera de forma sensible la satisfacción y el placer del paciente con su vida cotidiana. Se trata de un instrumento aplicable a todo tipo de pacientes independientemente del diagnóstico y tratamiento. Consta de 93 ítems y las áreas que explora son: Estado de salud, estado de ánimo, trabajo*, actividades del hogar*, tareas de clase/curso*, actividades de tiempo libre, relaciones sociales, actividades generales, satisfacción con la medicación y la satisfacción global. El marco de referencia temporal es la semana pasada. Esta escala ha sido validada para personas con esquizofrenia, esquizoafectivos y trastorno del ánimo (56).

Si nos centramos en las escalas creadas específicamente para valorar la calidad de vida en salud mental nos encontramos con la QLS, la CSCV y la LQoLP. La Escala de Calidad de Vida (Quality of Life Scale, QLS), originalmente, tal como sus autores (57), fue desarrollada para la evaluación de la disfunción del paciente debida al defecto esquizofrénico (58). Sin embargo, en la mayor parte de los estudios se aplica con la intención de determinar el nivel de calidad de vida del paciente. Consta de 21 ítems que se agrupan en las siguientes 4 factores: funciones intrapsíquicas, relaciones interpersonales, rol instrumental, uso de objetos comunes y actividades cotidianas. Se trata de una escala heteroaplicada que debe ser aplicada por un clínico entrenado en el uso de la entrevista semiestructurada. En la actualidad está bastante en desuso. Existe una versión en castellano, de la que no tenemos referencia de que se haya validado.

La LQoLP (Lancashire Quality of Life Profile) $(59,60)$ es un entrevista estructurada para valorar la calidad de vida de enfermos mentales crónicos, desa-

\footnotetext{
* A contestar por aquellos que ejerzan alguna de estas tareas. En el caso de las cuestiones sobre trabajo, contestar aunque se trabaje sin remuneración.
} 
ORIGINALES Y REVISIONES

rrollado por Oliver y cols (61) a partir de la Entrevista de Calidad de Vida de Lehman y que precisa aproximadamente de 30' en su cumplimentación. Evalúa las siguientes áreas: Trabajo/educación, tiempo libre, religión, aspectos económicos (finanzas), alojamiento, problemas legales y de seguridad ciudadana, relaciones familiares, relaciones sociales, salud y autoestima. Cada área se define en secciones, dónde en cada una se incluyen ítems de valoración objetiva y subjetiva. Además se añaden en la entrevista otras dos medidas de bienestar psicológico: la Affect Balance Scale de 10 ítems, a modo de indicador de salud mental y la Self-Esteem Scale, también de 10 ítems, que valora tanto la autoestima positiva como la negativa. El instrumento también valora el bienestar global. Incluye una evaluación de la calidad de vida basada en la observación y el conocimiento previo del caso por parte del entrevistador. No tenemos constancia de su validación, aunque existe traducción al castellano.

El Cuestionario Sevilla de Calidad de Vida (CSCV) $(62,63)$ es un instrumento diseñado para evaluar la calidad de vida del paciente esquizofrénico tal y como éste la percibe $(64,65)$. Consta de 59 ítems que se agrupan en 2 escalas: Aspectos favorables (satisfacción vital, autoestima y armonía) y aspectos desfavorables (alta de aprehensión cognitiva, pérdida de energía, falta de control interno, dificultad de expresión emocional, dificultad de expresión cognitiva, extrañamiento, miedo a la pérdida de control, hostilidad contenida y autoestima). Es una escala autoaplicada que tiene de marco de referencia temporal el momento actual. Esta escala ha sido validada en población española.

Por último, cabe destacar un cuestionario de calidad de vida específico para personas con trastorno mental severo diseñado por Baker e Intagliata (1982) (66). Esta escala de satisfacción con la vida es autoadministrada y consta de 15 ítems que miden aspectos como: lugar de residencia, barrio, comida, ropa, salud, relación con las personas que vive, con los amigos, con la familia, con otras personas, ocupación diaria, tiempo libre, entorno, servicios del barrio, situación económica y lugar de residencia respecto al hospital. Este cuestionario ha sido validado en castellano (67).

\section{Evaluación psicopatológica}

La irrupción de la enfermedad mental provoca multiplicidad de síntomas que emergen tanto en cualquiera de las fases de ésta, prodrómica- activa- residual como en las recidivas posibles. Con objeto de optimizar la intervención en el marco de la rehabilitación comunitaria, planificar e implementar un programa individualizado para cada sujeto, se utilizan instrumentos diseñados para regis- 
trar la sintomatología presente y las variaciones de ésta. Las diferentes escalas utilizadas proporcionan información estructurada sobre la presencia, modalidad y severidad existentes.

Las escalas más utilizadas para la valoración de la psicopatología son la Escala de Síndrome Positivo y Negativo para la Esquizofrenia (PANSS), la Escala Breve de Evaluación Psiquiátrica (BPRS), la Escala de Evaluación de la Actividad Global (EEAG clínica) y la Escala de Impresión Clínica Global (CGIesquizofrenia).

La PANSS (68) evalúa tres subescalas de psicopatología: síntomas positivos, negativos y generales. La PANSS constituye una escala validada que se creó con objeto de aumentar la rigurosidad en cuanto a establecer medidas tanto de la sintomatología positiva como de la negativa y otras graduaciones de la sintomatología presente en la Esquizofrenia.

Está traducida y validada al castellano por Peralta y Cuesta (69). La escala PANSS permite la clasificación categorial de la sintomatología presente en la Esquizofrenia y otros trastornos psicóticos. Aporta no exclusivamente la presencia y severidad sino también el grado de distorsión que puede ocasionar a la vida del sujeto la intensidad del trastorno mental.

Proporciona una evaluación cuantitativa y clasificatoria de la Esquizofrenia que se obtiene a través de una entrevista semiestructurada, para la existe un manual de entrenamiento realizado por Cuesta y Peralta. Las mediciones surgen sobre información sobre la entrevista, de la conducta observada, así como de la información proporcionada por la familia y los auxiliares.

La evaluación consiste en la suma de los ítems de la escala positiva, negativa, general y también se puede calcular la compuesta a partir de los positivos y negativos, valorando el grado de predominancia existente de un síndrome frente al otro. El análisis factorial pone de manifiesto la existencia de cuatro factores que explican el 52,3\% de la variancia total. Los cuatro factores responden a: Sintomatología Positiva, Negativa, Excitación y Depresión (70).

La Escala está formada por 30 ítems. La subescala positiva incluye: delirios, desorganización conceptual, comportamiento alucinatorio, excitación, grandiosidad, suspicacia - perjuicio y hostilidad. La subescala negativa incluye: embotamiento afectivo, retraimiento emocional, contacto pobre, retraimiento social, dificultad pensamiento abstracto, ausencia de espontaneidad y fluidez en la conversación y pensamiento estereotipado. Por último, la subescala general valora: preocupaciones somáticas, ansiedad, sentimientos de culpa, tensión motora, manierismos y posturas, depresión, retardo motor, falta colaboración, inusuales contenidos del pensamiento, desorientación, atención deficiente, ausencia de juicio e introspección, trastorno volición, control deficiente de impulsos, preocupación y evitación social activa. 
ORIGINALES Y REVISIONES

Cada uno de los 30 ítems valora en una escala de 7 puntos de evaluación, de menor a mayor gravedad.

La Escala Breve de Evaluación Psiquiátrica (BPRS) fue creada por Overall y Gorham (71) y evalúa los cambios sintomatológicos en pacientes psiquiátricos. Existe una versión castellana validada en Perú (72).

El objetivo que pretendía con ésta escala era poder realizar evaluaciones rápidas y eficientes de los cambios registrados en la sintomatología de los pacientes con enfermedad mental. Actualmente se utiliza como medida de gravedad y subtipificación en la Esquizofrenia.

Probablemente sea ésta escala de evaluación la más difundida en cuanto a uso en psiquiatría, originariamente constaba de 16 ítems a los que actualmente se le han añadido dos aunque también existe una versión ampliada a 24 ítems.

Se creó fundamentalmente para ser utilizada en cuadros de Esquizofrenia pero también comprende síntomas de Depresión. Ofrece la gran ventaja de poder documentar los cambios siguiendo un método taquigráfico muy fácil. Se recomienda se evalúe la presencia y el grado para cada ítem según el estado del paciente a la fecha de la entrevista y durante los últimos tres días. Deben valorarse: ansiedad psíquica, hostilidad, estado de sospecha, conducta alucinatoria, pensamientos inhabituales y afectos bloqueados o inadecuados.

La escala es cuantitativa se creó con el objeto único de evaluar el cuadro clínico actual y se realiza en el contexto de entrevista semiestructurada. Proporciona una puntuación global y puntuaciones en dos apartados: síntomas positivos y síntomas negativos. Los puntos de corte obtenidos mediante escala Likert proporcionan tres posibilidades: ausencia de trastorno, trastorno leve o caso probable y trastorno grave o caso cierto.

La Escala de Impresión Clínica Global (CGI) para esquizofrenia fue validada por Josep Maria Haro et al (73). La CGI se refiere a la impresión global del paciente y la evaluación requiere experiencia clínica en el síntoma que se evalúa. Consta de dos subescalas que evalúan: gravedad del cuadro clínico y mejoría del cuadro debida a intervenciones terapéuticas. Ambas escalas constan de un único item. Existen versiones heteroaplicadas o autoaplicadas. La CGI es una escala descriptiva que proporciona información cualitativa sobre la gravedad del cuadro y sobre el cambio experimentado por el paciente con respecto al estado basal. El concepto de mejoría se refiere a la distancia existente entre el estado actual del paciente y el que se registraba al inicio del tratamiento, puede completarse únicamente durante o después del mismo.

Las categorías presentan una gradación que va desde "sin enfermedad " a "extremadamente grave". 
ORIGINALES Y REVISIONES

\section{Carga Familiar}

El desarrollo de la psiquiatría comunitaria, tuvo como consecuencia una desinstitucionalización de las personas con enfermedades mentales que permanecían ingresadas en los hospitales. Durante este proceso numerosas familias se han hecho cargo de su familiar enfermo y de su cuidado. En el proceso de rehabilitación psicosocial la familia tiene un papel importante como facilitadota del cambio, por este motivo es necesario evaluar cómo la familia puede ayudar en él. Por otro lado, el impacto o carga familiar que genera el cuidado de una persona con un trastorno mental severo debe ser evaluado puesto que facilita al terapeuta a intervenir en aquellos aspectos que afecten más negativamente la relación con la persona que ejerce de cuidadora.

Existen pocos instrumentos que valoren la carga familiar de personas con trastorno mental severo. De ellos, los más utilizados son el "Social Behaviour Assessment Schedule" (SBAS) (74); el "Experience of Caregiving Inventory (ECI)"(75), el "Involvement Evaluation Questionnaire" (IEQ) (76) y el "The Family Burden Interview Schedule - Short Form (FBIS-SF)" (77).

De todos ellos, solamente dos están validados al castellano: el IEQ y el FBIS-SF que en castellano se denomina ECFOS-II. El EIQ, fue validado por el grupo EPSILON y consta de cinco subescalas (tensión, supervisión, preocupación, impulsos y otros ítems no incluidos) compuestas por un total de 33 ítems. Este cuestionario mide la carga familiar, teniendo en cuenta la dimensión objetiva, es decir, evaluando las frecuencias de conducta de ayuda o las preocupaciones del familiar.

El ECFOS-II ha sido validado por el grupo PSICOST (78). La ventaja respecto al anterior es que permite medir la carga o impacto familiar objetiva pero también la subjetiva, los aspectos positivos de la carga familiar y también el desglose en costes que generan los cuidados. La entrevista está compuesta por diferentes módulos que evalúan las dimensiones de la carga familiar: la ayuda proporcionada en las actividades de la vida diaria del paciente; la contención realizada de los comportamientos alterados; un listado de los gastos económicos; el impacto sobre la vida del cuidador; los motivos de preocupación por el paciente; la ayuda disponible; los efectos percibidos sobre su salud y las repercusiones globales experimentadas tanto a nivel individual como familiar. Se evalúa la presencia de carga en cada una de las preguntas de los dos primeros módulos, y si existe carga se valora la frecuencia y la preocupación que esta área genera.

De los instrumentos sobre carga familiar que no están validados al castellano, destaca el SBAS y el ECI, que a pesar de no estar validados están traducidos y se utilizan en algunos contextos. El SBAS ha sido adaptado por Otero, Navascues y Rebolledo (79), es una entrevista semiestructurada diseñada para evaluar 
ORIGINALES Y REVISIONES

a través de 7 secciones diferentes el efecto del comportamiento del sujeto en sus parientes y el estrés que éste produce en el informante, pero no permite obtener puntuaciones globales. Evalúa por tanto el espectro psicopatológico y el funcionamiento social, y también carga subjetiva y objetiva causada por la enfermedad. Además de aportar información del apoyo y demandas q le genera la familia. También se toman en cuenta algunas variables: acontecimientos vitales y apoyos externos.

El ECI, en cambio, es un instrumento autoaplicado que intenta, a través de 66 ítems, explorar la evaluación que los cuidadores realizan de los aspectos positivos y negativos de la experiencia de cuidar de una persona con un trastorno mental.

Por último hablamos de la Escala de Sobrecarga del Cuidador de Zarit (80). Esta escala está diseñada para evaluar la sobrecarga de los cuidadores de los pacientes con demencia desde la teoría general del estrés. Consta de 22 ítems, autoaplicados, que evalúan las repercusiones negativas sobre determinadas áreas de la vida asociadas a la prestación de cuidados: salud física, salud psíquica, actividades sociales y recursos económicos. Esta escala ha sido traducida y adaptada a la población española, en algunas situaciones se ha utilizado para la evaluación de carga familiar en personas con una patología mental (81).

\section{Conclusiones}

A lo largo de este artículo hemos dado constancia de la existencia de numerosas escalas de evaluación que resultan útiles para la detección de las áreas de intervención en rehabilitación psicosocial. Para poder analizar estos instrumentos optamos por establecer seis grandes áreas de evaluación: evaluación general en rehabilitación psicosocial, evaluación de necesidades, evaluación de discapacidad y el funcionamiento psicosocial, calidad de vida, evaluación psicopatológica y carga familiar. La evaluación de la efectividad del abordaje en la recuperación de las enfermedades mentales de larga evolución debe ser uno de los estándares a los que deben aspirar todos los servicios de rehabilitación. A pesar de que existen numerosos instrumentos, no todos ellos están validados en población diana.

En la revisión llevada a cabo, específicamente para la evaluación general de la persona con trastorno mental severo están validadas las escalas las siguientes escalas: HONOS y la REHAB (generales), la CAN y la 2-COM (necesidades), la LSP y la SFS (para la discapacidad y el funcionamiento psicosocial ), la CSCV, la SF-36, la EQ-5D y la Q-LES-Q (calidad de vida), la PANSS y la CGI (evaluación psicopatológica) y la IEQ y la ECFOS-II (carga familiar). 
En la práctica clínica se siguen utilizando escalas que no han sido validadas, por ser las únicas que valoran un área concreta. Sin embargo nos tendríamos que plantear la validación para obtener información más fiable.

\section{Lineas de futuro}

En la actualidad, y ante la inquietud de trabajar bajo los parámetros de evidencia, eficacia y eficiencia, existe una gran inquietud para la validación de escalas. Esta línea de trabajo es básica para mejorar la fiabilidad de la información obtenida y ajustar los resultados a las características específicas de la población que recibe tratamientos de rehabilitación psicosocial. Desarrollar escalas más específicas para la población y la realidad del enfermo mental crónico en nuestro contexto es imprescindible, por ejemplo, en el área de las habilidades sociales, aún siendo una de las áreas con demostrada efectividad en la rehabilitación, no disponemos de escalas específicas para su adecuada evaluación.

Sería interesante poder disponer de un protocolo de evaluación consensuado en el área de la rehabilitación, ello permitiría evaluar las intervenciones de forma sistemática, utilizando un lenguaje común para todos los profesionales, y mejoraría la objetivación de la efectividad de los programas de intervención. Actualmente, a nivel cualitativo la mejoría de los pacientes con patología mental severa que reciben tratamiento especializado en rehabilitación psiquiátrica es notable, reflejándose en una disminución de las recaídas, aumento de la calidad de vida, disminución de la sobrecarga familiar. Sin embargo, la evaluación cuantitativa y cualitativa es escasa en el ámbito de la rehabilitación psiquiátrica siendo necesario implementar estos parámetros en la práctica clínica diaria. La evaluación entendida como acción integrada en la práctica diaria nos permitiría ajustar el rigor de las intervenciones realizadas en las áreas descritas anteriormente: necesidades, funcionamiento psicosocial-discapacidad-actividades de la vida diaria, calidad de vida, psicopatología, y carga familiar.

Otro punto importante a destacar como línea de futuro es la evaluación en las primeras fases de las enfermedades mentales crónicas, dada su importancia para evitar la iatrogenia y la cronificación. La utilización de escalas y el diseño de protocolos de evaluación son indispensables. 
ORIGINALES Y REVISIONES

\section{BIBLIOGRAFÍA:}

(1) Liberman RP, Kopelwicz A. Un Enfoque empírico de la recuperación de la esquizofrenia: definir la recuperación e identificar los factores que pueden facilitarla. Rehabilitación Psicosocial 2004; 1 (1):12-29.

(2) Gumley A Et Al. Early intervention for relapse in schizophrenia: Impact of cognitive behavioural therapy on negative beliefs about psychosis and self-esteem. Br J Clin Psychol. 2006 Jun;45(Pt 2):247-60.

(3) Garcia M et AL. ¿Está demostrada la rehabilitación psicosocial en la esquizofrenia? Controversias actuales en las diferentes intervenciones y enfoques. Aula Médica Psiquiátrica 2001; 1.

(4) Fernández Ballesteros, R. Introducción a la evaluación psicológica. Madrid: Pirámide, 1999.

(5) Fischer EP ET AL. Schizophrenia outcomes module (SCHIZOM) $1996 \mathrm{http}: / /$ measurementexperts.org/instrument/instrument_reviews.asp?detail=73. Obtenido el 4 de abril de 2007.

(6) WING JK ET AL. Health of the Nation Outcome Scales (HoNOS): Research and development. British Journal of Psychiatry 1998; 172: 11-18.

(7) Uriarte JJ et al. Presentación de la traducción al castellano de la escala HoNOS (Health of the Nation Outcome Scales). Psiquiatría Pública 1999; 11 (4): 93-101.

(8) BaKer R, Hall J. Rehabilitation Evaluation of Hall and Baker (REHAB), Aberdeen, Escocia: Vine Publishing, 1983.

(9) BAKER R, Hall J. REHAB: A new assessment instrument for chronic psychiatric patients. Schizophrenia Bulletin 1988; 14 (1), 97-111

(10) Jiménez R, Vazquez AJ, Cecilia L. REHAB: Características psicométricas de una versión española, Psiquis 1994; 15 (5): 33-40.

(11) BREWIN CR, WING JK. Principles and practice of measuring needs in the longterm mentally ill: the Needs for Care Assessment. Psychological Medicine, 1987, 17: 971-981.

(12) Marshall M. How should we measured need? Philosophy, psychiatry and psychology 1994; 1: 27-36.

(13) Phelan M et al. The Camberwell Assessment of Need (CAN): the validity and reliability of an instrument to assess the needs of people with severe mental illness. British Journal of Psychiatry 1995; 167: 589-595.

(14) Rosales C et al. Fiabilidad del instrumento de evaluación de necesidades Camberwell (CAN). Actas Españolas de Psiquiatría 2002; 30, 2: 99-104.

(15) Hansson L, Bjorkman T, Svensson B. The assessment of needs in psychiatric patients. Interrrater reliability of the Swedish version of the Camberwell Assessment of Needs instrument and results from a cross sectional study. Acta Psychiatrica Scandinavica, 1995, 92: 285-293.

(16) RugGeri M ET AL. The italian version of the Camberwell Assessment of Need (CAN), an interview for the identification of needs of care. Epidemiologia e Psichiatria Sociale 1999; 8: 135-167.

(17) KNUdSEN HC ET AL . Translation and cross-cultural adaptation of outcome measurement for schizophrenia, British Journal of Psychiatry 2000; 177 (suppl 39, s8-s14).

(18) Mc Crone P et Al. Reliability of the Camberwell Assessment of Need- European version. British Journal of Psychiatry 2000; 177 (39): s34-40.

(19) Andresen R, CAputi P, OAdes LG. Interrater reliability of the Camberwell Assessment 
ORIGINALES Y REVISIONES

of Need Short Appraisal Schedule. Australian and New Zeland Journal of Psychiatry 2000; 34: 856861.

(20) Slade M et Al. Routine clinical outcome measures for patients with severe mental illness:CANSAS and HONOS. British Journal of Psychiatry 1999; 174: 404-408.

(21) VAN Os J ET AL. 2- COM: an instrument to facilitate patient-professional communication in routine clinical practice. Acta Psychiatrica Scandinavica 2002; 106: 446-452.

(22) Frey WD. Functional assesment in the 80s: A conceptual enigma, a technical challenge. En Halpern A, Furher M (eds). Functional Assesment in Rehabilitation. Nueva York: Brooke Publishing 1984.

(23) Organización Mundial de la Salud. WHO-DAS II (http://www.who.int/icidh/whodas) 20 de diciembre de 2006

(24) JAnCA A ET AL. The World Health Organization Short Disability Assessment Schedule (WHO DAS-S): a tool for the assessment of difficulties in selected areas of functioning of patients with mental disorders. Soc. Psychiatry Psychiatr. Epidemiol 1996; 31: 349-54.

(25) Schubart C Et Al. Measurement of social disability in a schizophrenic patient group. Definition, assessment and outcome over 2 years in a cohort of schizophrenic patients of recent onset. Soc Psychiatry. 1986; 21(1):1-9.

(26) Montero I et Al. Adaptación española del DAS II (Disability Assessment Schedule). Psiquis 1988; 175: 17-22.

(27) Montero I, Gomez-Beneyto M. Funcionamiento social medido por medio del DAS II en una muestra de pacientes esquizofrénicos seguidos a lo largo de dos años (Evaluation of social adjustment in schizophrenic patients with the DAS-II: two years follow-up). Psiquis 1993; vol. 14, no10: 27-33

(28) Endicott J ET AL. The Global Assessment Scale: a procedure for measuring overall severity of psychiatric disturbance. Archives of General Psychiatry, 1976, 33: 766-771

(29) Rosen A, Had-zi.Pavlovic D, Parker G. "Thre Life Skills Profile: A measure assessing function and disability in schizophrenia", Schizophrenia Butlletin 1989; 15: 325-337.

(30) Bulbena A, Fernández de Larrinoa P, Domínguez A. Adaptación Castellana de la Escala LSP. Perfil de Habilidades de la Vida Cotidiana. Actas Luso-Esp. Neurol. Psiquiatr 1992; 20, 2 (51-60).

(31) Fernández de Larrinoa P, Bulbena A, Domínguez A. Estudio de Fiabilidad, Validez y Consistencia Interna de la Escala LSP. Perfil de Habilidades de la Vida Cotidiana. Acatas Luso-Esp. Neurol. Psiquiatr. 1992; 20, 2: 71-75.

(32) O'Driscoll, C And LefF, J. The Taps Project.8: design of the research study on the long stay patients. Br. J. Psychiatry 1993; 162 (supl 19): 18-24.

(33) Wallace, C.J. Social skills training in psychiatric rehabilitation: Recent Findings. Social Psychiatry and Psychiatric Epidemiology 1997; 28: 5-10.

(34) Fernández Larrinoa P et AL. Estudio de fiabilidad y validez de la escala ILSS (Escala de Habilidades para una Vida Independiente). Manuales de Psicología 1994; VI.

(35) Birchwood M. et AL. The Social Functioning Scale. The development and validation of a new scale of social adjustment for use in family intervention programmes with schizophrenic patients. Br. J. Psyachiatry, 1990; 157: 853-859.

(36) Torres A, Olivares JM. Validation of the Spanish version of the Social Functioning Scale. Actas espa.psiquiatria 2005 Jul-Aug; 33(4): 216-20. 
ORIGINALES Y REVISIONES

(37) Caballo VE. Evaluación y entrenamiento de las Habilidades Sociales: una estrategia multimodal. Tesis doctoral, Universidad Autónoma de Madrid. 1987.

(38) Caballo VE. La multidimensionalidad conductual de las Habilidades Sociales: Propiedades psicométricas de una medida de autoinforme, la EMES-M. Psicología Conductual 1993; 1: 221-231.

(39) Caballo VE, Ortega AR. La Escala multidimensional de Expresión Social: algunas propiedades psicométricas. Revista de Psicología General y Aplicada 1989; 42: 215-221.

(40) Cirici R, et AL. Competencia Social en la Esquizofrenia: Discrepancias por sexos. Cuadernos de Medicina Psicosomática 1993; 27: 6-11. ISSN. 1132-0273.

(41) Bel MT Et AL. Revisión sobre las autoevaluaciones de la interacción social en sujetos esquizofrénicos. Revista de rehabilitació psicosocial integral en la comunitat i amb la comunitat 1997; 1-2 (2-3): 15-19. Copyright 1995 Centre de Documentació, Docència i Recerca ARAPDIS.

(42) Gambrill ED, Richey CA. An assertion inventory for use in assessment and research. Behavior Therapy 1975; 6, 550-561.

(43) Organización Mundial de la Salud. Quality of Life Assessment. An Annotated Bibliography. WHO (MNH/PSF/94.1), Geneva 1994.

(44) Malm U, May PR, Dencker SJ. Evaluation of the quality of life of the schizophrenic outpatient: a checklist. Schizophr Bull. 1981; 7(3): 477-87.

(45) Barcia D. Prólogo. En: Bobes J, González M.P, Bousoño M. Calidad de vida en las esquizofrenias. Barcelona: J.R. Prous Editores, 1995.

(46) Ware JE JR, Sherbourne CD. The MOS 36-item short-form health survey (SF-36). I. Conceptual framework and item selection. Med Care 1992; 30(6): 473-83.

(47) Alonso J.; Prieto L.; Antó J.M. La versión española del SF-36 Health Survey (Cuestionario de Salud SF-36): Un instrumento para la medida de los resultados clínicos. Med Clin (Barc) 1995; 104: 771-776.

(48) Badía X et AL. La Medida de la Salud. Guía de escalas de medición en español. Barcelona: PPU, 1996. 108- 115.

(49) BADÍA X, ET AL. Using the EuroQoL 5- D in the Catalan general population: Feasibility and construct validity. Qual Life Res, 1998; 7: 311-322.

(50) Badía X., ET AL. The Spanish version of EuroQoL: A description and its applications. European Quality of Life scale. Med Clin (Barc) 1999; 112 (Supl 1): 79-85.

(51) EuroQoL Group. EuroQoL- a new facility for the measurament of health-related quality of life. Health Policy 1990;16: 199-208.

(52) Organización Mundial de la Salud (WHOQOL Group). Measuring quality of life. The development of the Word Health Organization Quality of Life Instrument (WHOQOL-100). Genève: OMS, 1998.

(53) Lucas Carrasco, R. Versión española del WHOQOL. Madrid: Ergón, 1998. Malm U, May P, Dencker SJ. Evaluation of the Quality of Life of the Schizoprenia Outpatient: A Checklist. Schizophrenia Bulletin 1981; 7 (3): 477-487.

(54) Endicott J ET AL. Quality of Life Enjoyment and Satisfaction Questionnaire: A new measure. Psychopharmacol Bull 1993; 29: 321-326.

(55) BISHOP SL ET AL. Refining quality of life: Validating a multidimensional factor measure in the severe mentally ill. Qual Life Res 1999; 8: 151-160.

(56) Ritsner M ET AL. Validity of an abbreviated quality of life enjoyment and satisfaction 
questionnaire (Q-LES-Q-18) for schizophrenia, schizoaffective, and mood disorder patients. Qual Life Res. 2005 Sep;14(7):1693-703.

(57) Heinrichs DW, Hanlon TE, Carpenter WT. The Quality of Life Scale: An instrument for rating the schizophrenia deficit syndrome. Schizoprhr Bull 1984; 10: 388-399.

(58) Rodríguez Fornells A, ET AL. Validez de constructo de la escala de calidad de vida en la esquizofrenia (QLS). Barcelona: Sexto Congreso Associations of European Psychiatrists, 1992.

(59) VÁZqueZ-BARQuero JL ET AL. Desarrollo de la versión española del perfil de calidad de vida de Lancashire (LQOLP). Archivos de Neurobiología 1997; 60: 125-139.

(60) Gaite L ET AL. Quality of life in schizophrenia: development, relizability and internal consistency of the Lancashire Quality of Life Profile-European versión. EPSILON Study 8. European Psychiatric Services: Inputs Linked to outcome domains and needs. Br J Psychiatry 2000; 177 (suppl 39): 49-54.

(61) Oliver JP ET AL. Measuring the quality of life of severely mentally ill people using theLancashire Quality of Life Profile. Soc Psychiatry Psychiatr Epidemiol 1997 Feb; 32(2): 76-83

(62) Giner J Et AL. Calidad de vida en enfermos esquizofrénicos. Desarrollo de un instrumento español para su evaluación: el Cuestionario "Sevilla". Anales de Psiquiatría 1995; 11: 313-319.

(63) Giner J et al. Desarrollo del Cuestionario Sevilla de Calidad de Vida (CSCV). Actas Luso Esp. Neurol. Psiquiatr. Cienc. Afines 1997; 25 (supl 2): 11-23.

(64) Seonane J. Estilos y tipos de calidad de vida en el Cuestionario Sevilla. Actas Esp Psiquiatri 1999; 27: 245-249.

(65) Giner J ET AL. Experiencia subjetiva y calidad de vida en esquizofrenia. Actas Esp. Psiquiatr 2001; 29: 233-242.

(66) BAKER F, INTAGLiata J. Quality of life in the evaluation of community support systems. Evaluation and program planning 1982, 5: 69-79.

(67) CARLSON J. Estudio de la calidad de vida de personas con esquizofrenia seguimiento a 7 años. Tesis doctoral. 2007. Universidad Autónoma de Barcelona.

(68) KAY SR, Opler LA, Fiszbein A. The positive and negative symptom scale (PANSS). Rating manual. Social and Behavioural Sciences Documents 1986; 17: 28-29.

(69) Peralta V, Cuesta JM. Validación de la escala de síndromes positivo y negativo (PANSS) en una muestra de esquizofrénicos españoles. Actas Luso-Españolas de Psiquiatría 1994; 22: 171-177.

(70) KaY SR, SEvY S. Pyramidical model of schizophrenia.Schizophr Bull. 1990; 16(3): 537-45.

(71) Overall JF, Gorham, DR. The Brief Psychiatric Rating Scale. Psychol. Rep. 1962; 10: $799-812$.

(72) Vasquez GA et AL. Validación de la brief psychiatric rating scale (bprs) en el Perú. Revista de neuro-psiquiatría del Perú 2001; Tomo LXIV Septiembre, $N^{\circ} 3$.

(73) Haro JM et AL. The Clinical Global Impression-Schizophrenia scale: a simple instrument to measure the diversity of symptoms present in schizophrenia. Acta Psychiatrica Scandinavica Suppl. 2003; 416: 16-23.

(74) Platt S et Al. The Social Behaviour Assessment Schedule (SBAS): rationale, contents, scoring and reliability of a new interview schedule. Soc Psychiatry 1980;15:43-55.

(75) Joyce J; Leese M; SzMuKLer G. The Experience of caregiving inventory: further evidence. Soc. psychiatry psychiatr. Epidemiol, 2000, 35 (4): 185-189.

(76) WIJNGAARDEN B ET AL. Caregiving in schizophrenia: development, internal consistency 
and reliability of the Involvement Evaluation Questionaire-European Version. British Journal of Psychiatry, 2000, 39, s21-27.

(77) Tessler R.C.; Gamache G.M. The Family Burden Interview Schedule- Short Form (FBIS-SF). En: TeSSLER R.C.; Gamache G.M., Cambridge: Human Services Research Institute, 1995; p.110-112.

(78) Vilaplana M et al. Validación en población española de la entrevista de carga familiar objetiva y subjetiva (ECFOS-II) para familiares de personas con esquizofrenia. Actas Españolas de Psiquiatría, 2007;35 (6): 372-81.

(79) Otero V, Navascues D, Rebolledo S. Escala de Desempeño Psicosocial. Adaptación Española del SBAS (Social Behavour Assessment Schedule). Psiquis 1990; XI: 395-401.

(80) Zarit SH, Rever KE, Bach-Peterson J. Relatives of the impaired elderly: Correlates of feeling of burden. Gerontologist 1980; 20: 649-654.

(81) Martín M et AL. Adaptación para nuestro medio de la Escala de Sobrecarga del Cuidador (Caregiver Burden Interview) de Zarit. Revista de Gerontología 1996; 6: 338-346.

* EMMA CASAS: Dra en Psicopedagogía. Servicio de Rehabilitación Psiquiátrica Intensiva. Sant Joan de Déu-Serveis de Salut Mental.

MARIA JOSE ESCANDELL: Psicóloga Clínica. Centro de Día de Cerdanyola. Sant Joan de Déu-Serveis de Salut Mental.

MARIA RIBAS: Psicóloga Clínica. Unidad Terapéutica de Justicia Juvenil Els Til.lers. Sant Joan de DéuServeis de Salut Mental.

SUSANA OCHOA: Dra en Psicología. Unidad de Investigación. Sant Joan de Déu-Serveis de Salut Mental. Fundació Sant Joan de Déu. CIBERSAM.

Correspondencia: Susana Ochoa Güerre. Unitat de Recerca Sant Joan de Déu-SSM.

C/ Pujades, 42. Tel : 936406350 ext : 2356 Correo electrónico: sochoa@sjd-ssm.com

Agradecimientos:

Financiado por el Fondo de Investigación Sanitaria, Instituto de Salud Carlos III, CIBERSAM.

** Recibido: 11/02/2008 